\title{
Atomic water channel controlling remarkable properties of a single brain microtubule: Correlating single protein to its supramolecular assembly
}

\author{
Satyajit Sahu ${ }^{\mathrm{a}}$, Subrata Ghosh ${ }^{\mathrm{a}}$, Batu Ghosh ${ }^{\mathrm{c}}$, Krishna Aswani ${ }^{\mathrm{d}}$, Kazuto Hirata ${ }^{\mathrm{b}}$, \\ Daisuke Fujita ${ }^{a}$, Anirban Bandyopadhyay ${ }^{\mathrm{a}, *}$ \\ a Nano Characterization Unit, National Institute for Materials Science, 1-2-1 Sengen, Tsukuba, Ibaraki 305-0047, Japan \\ ${ }^{\mathrm{b}}$ Vortex Dynamics Group, National Institute for Materials Science, 1-2-1 Sengen, Tsukuba, Ibaraki 305-0047, Japan \\ ${ }^{\mathrm{c}}$ Materials and Nano-architectronics, National Institute for Materials Science, 1-2-1 Sengen, Tsukuba, Ibaraki 305-0047, Japan \\ d Surface Characterization Group, Nano Characterization Unit Advanced Key Technologies Division, National Institute for Materials Science, 1-2-1 Sengen, \\ Main Bldg, Room-815 Tsukuba, 305-0047, Japan
}

\section{A R T I C L E I N F O}

Article history:

Received 7 January 2013

Received in revised form

20 February 2013

Accepted 21 February 2013

Available online 15 March 2013

Keywords:

Microtubule

Tubulin protein

Scanning tunneling microscopy

Four probe electronic device

Atomic force microscopy

Resonance spectroscopy

\begin{abstract}
A B S T R A C T
Microtubule nanotubes are found in every living eukaryotic cells; these are formed by reversible polymerization of the tubulin protein, and their hollow fibers are filled with uniquely arranged water molecules. Here we measure single tubulin molecule and single brain-neuron extracted microtubule nanowire with and without water channel inside to unravel their unique electronic and optical properties for the first time. We demonstrate that the energy levels of a single tubulin protein and single microtubule made of 40,000 tubulin dimers are identical unlike conventional materials. Moreover, the transmitted ac power and the transient fluorescence decay (single photon count) are independent of the microtubule length. Even more remarkable is the fact that the microtubule nanowire is more conducting than a single protein molecule that constitutes the nanowire. Microtubule's vibrational peaks condense to a single mode that controls the emergence of size independent electronic/optical properties, and automated noise alleviation, which disappear when the atomic water core is released from the inner cylinder. We have carried out several tricky state-of-the-art experiments and identified the electromagnetic resonance peaks of single microtubule reliably. The resonant vibrations established that the condensation of energy levels and periodic oscillation of unique energy fringes on the microtubule surface, emerge as the atomic water core resonantly integrates all proteins around it such that the nanotube irrespective of its size functions like a single protein molecule. Thus, a monomolecular water channel residing inside the protein-cylinder displays an unprecedented control in governing the tantalizing electronic and optical properties of microtubule.
\end{abstract}

(c) 2013 Elsevier B.V. All rights reserved.

\section{Introduction}

In spite of incredible claims, the carbon nanotube could not revolutionize the industry due to complicacy in isolating metallic and semiconducting nanotube, and the DNA adventure (Dekker and Ratner, 2001; Fink and Schönenberger, 1999; Rakitin et al., 2001; Storm et al., 2001; Zhang et al., 2002) turned critical due to its extreme conformational-fluctuations on the atomic scale. The $25 \mathrm{~nm}$ wide and from $200 \mathrm{~nm}$ to $25 \mu \mathrm{m}$ long microtubule nanotube stores cellular dynamics codes as doped drugs inside its main constituent tubulin protein similar to ATGC that stores DNA's genetic code. Nature has a catalog of microtubule's cellular code, in all eukaryotes, plants, animals, fungi and Protista kingdom for 3.5 billion years. It forms a complex network inside neurons and living cells controlling

\footnotetext{
* Corresponding author. Tel.: +81298592167.

E-mail addresses: anirban.bandyo@gmail.com, anirban.bandyopadhyay@nims.go.jp (A. Bandyopadhyay).
}

fundamental life functions via massively parallel and hierarchical information processing (Barabási and Albert, 1999; Butts, 2009; Gerhart et al., 1997; Moriya et al., 2001; Song et al., 2005; Strogatz, 2001). Since single tubulin and microtubule properties were never studied extensively, here we cater state-of-the-art technologies to unravel the electronics and information processing in these systems (Mange and Tomassini, 1998; Sipper, 2002; Teuscher et al., 2003; Zhang and Gao, 2012). As microtubules are dipped into an extremely noisy cellular soup (Braun et al., 2003; Roberts et al., 2011; Shibata and Ueda, 2008; Szendro et al., 2001a, 2001b), the properties studied therein contain artifacts, while noise-free bio-material studies are irrelevant to real bio-systems (Roberts et al., 2011). Yet, microtubule is a rigid elastic string unlike DNA and its composition of lattice mixtures is many folds more resourceful than carbon nanotube with no isolation issues-a prime candidate for the state-of-the-art investigations to unravel its embedded nanotechnologies.

The naturally produced drug molecules were automatically doped inside the tubulin protein to add unique properties to the 
microtubule while keeping the original properties intact. During design and construction of microtubule for a particular species following this route (Nielsen et al., 2010, 2006; Redeker et al., 2004), the microtubule structure remained unchanged. The origin of this flexibility is unknown. Moreover, the fusion of DNA-like coding via drug-molecules and carbon nanotube like modulation of property by changing lattice parameters requires identification of its true nano-material class. Consequent theoretical predictions of its remarkable properties (Sahu et al., 2011) were not verified experimentally. In this first comprehensive documentation, we underpin both the fundamental and the applied potentials of this nanotube. We compare single tubulin and microtubule's properties when water channel resides in its core and then after releasing the water in a controlled manner. The water channel couples helically wrapped tubulins such that even though microtubule is a complex composition of several distinct structural symmetries only the single tubulin property defines the microtubule property.

Protein is a single chain polymer, but folds into various patterns, called secondary structures; switching of these structures into an astronomically large number of combinations is restricted via allowed and blocked symmetries. Tubulin protein has two parts, $\alpha$ and $\beta$, both appear similar, connected face-to-face, see Fig. 1a. They assemble in a hexagonal close packing into a 2D sheet which folds into a hollow cylinder wrapped around a water channel (see Fig. 1a).

\section{Results and discussion}

\subsection{Identical energy levels of tubulin protein and microtubule}

Since combined excitation emission spectroscopy (CEES) provides fluorescence as a function of excitation and emission, the exact peak locations are identified, from which the allowed energy-level transitions in tubulin protein and microtubule were calculated (Fig. 1b). By density-variation-CEES-study, the threshold density $60 \mu \mathrm{M} / \mathrm{ml}$ is determined at which tubulin proteins and microtubules start interacting with each other, synchronously. So tubulin and microtubule solution were kept at a very low density $(<10 \mu \mathrm{M} / \mathrm{ml}$ ) (Dierolf and O'Donnell, 2010; Dierolf and Koerdt, 2000). Due to synchronization, fluorescence intensity oscillates periodically; onset of such oscillation in tubulin solution is shown in Fig. 1c. For microtubule solution, periodic oscillation disappears at lower than its threshold density (Fig. 1d). Identical energy level transitions of a single tubulin dimer and microtubule (Fig. 1b right) are the outcome of an unprecedented phenomenon. The remarkable fact that 30,000-40,000 dimers assemble into microtubule without changing the fundamental energy levels depicts that the energy levels of all tubulins interact but do not modify the levels in the polymer form. Band theory of metal, insulator or semiconductor is not applicable here, since two distinct transitions in Fig. 1b, eventually converge to the same levels so that emissions are always the same irrespective of the energy absorption in a single

a

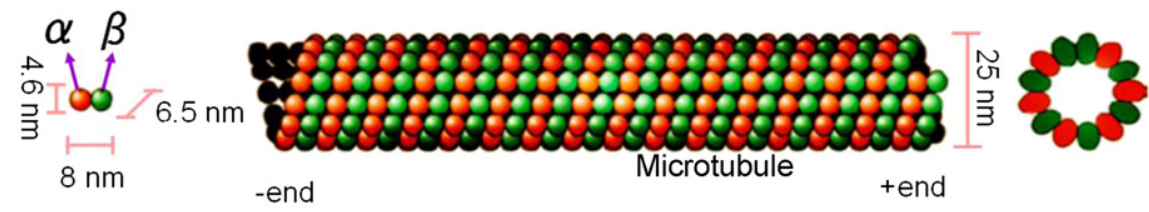

b
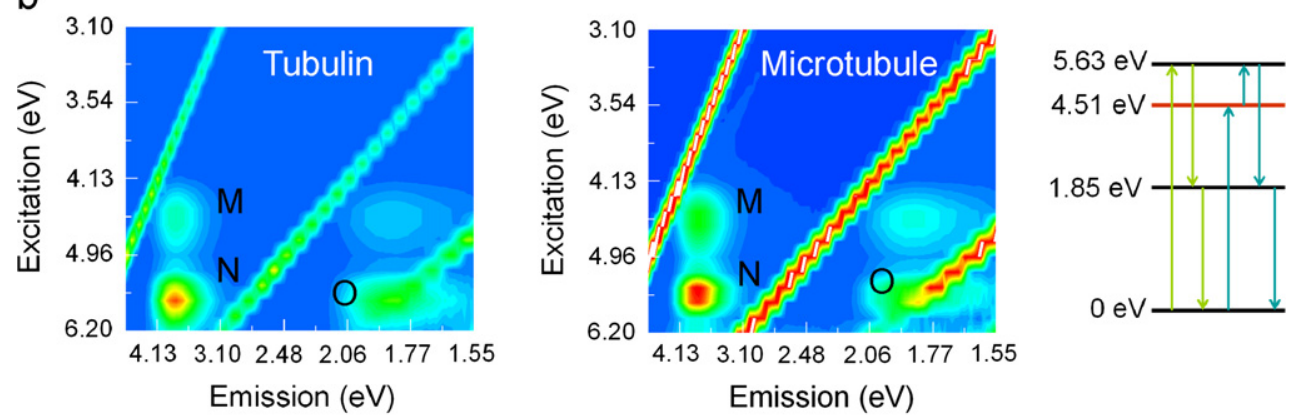

C

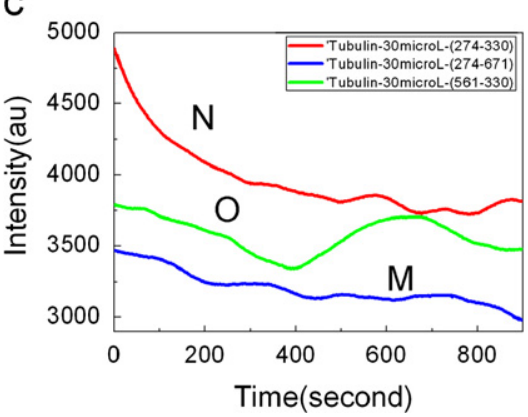

d

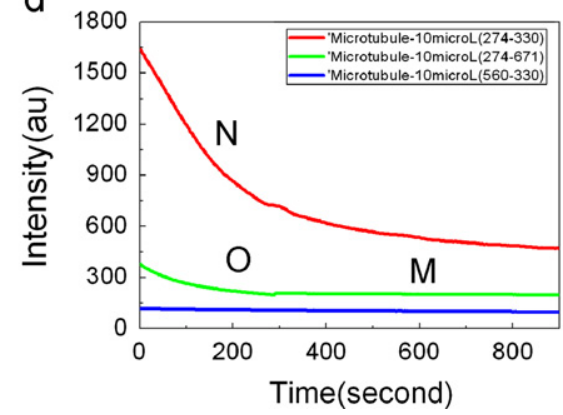

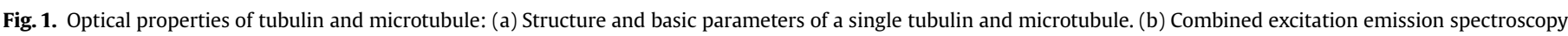

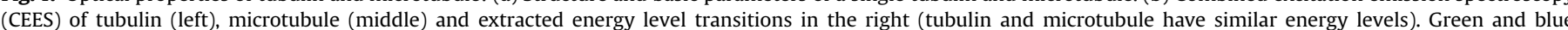

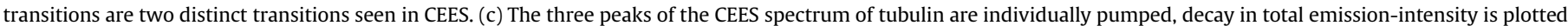

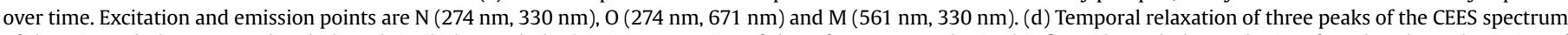

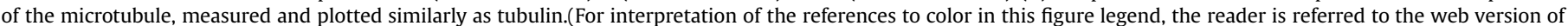
this article.) 
tubulin or microtubule. This behavior argues microtubule and tubulin as strong candidates for the spontaneous noise alleviation.

\subsection{Binary condensation of vibration and non-linear ultra-fast relaxation}

The optical study shows that external energy pumped into different levels converge into one similar to the energy condensation (Mesquita et al., 1993; Moskalenko et al., 1980; Rotaru et al., 1999), one could alternately model this behavior with fractured band structure (Grigor'kin and Dunaevskiǐ, 2007; Michalski and Mele, 2008; Prodan and Prodan, 2009). The temporal relaxations for three fluorescence peaks for $900 \mathrm{~s}$ suggest that for tubulin and microtubule, even intensity variation follows the same trend; in the absence of synchronization, it is an exponential decay process. Microtubule's synchrony is a profoundly documented phenomenon (Ahmad et al., 1994; Carlier et al., 1987), an optical study suggests that tubulin's global synchrony-behavior encompassing all proteins is responsible for the observed properties of proteinbuilt polymer microtubule. In other words, the incredible microtubule properties are encoded in the structure of the tubulin protein.

Fig. 2a shows that increasing the intensity of LASER power increases the emission linearly for tubulin. Therefore, neither single tubulin-dynamics survives for long, nor does it demonstrate any non-linear properties. However, similar studies with microtubule show that the emission intensity increases non-linearly, which means that the pumped energy vibrates all tubulins in the microtubule to a particular energy level that does not allow absorption of energy from outside, eventually, microtubule emits entire energy imparted to it. Literatures argue that non-linearity suggests optical cavity (Agarwal et al., 2005; Jelínek and Pokorný, 2001; Oulton et al., 2008; van Vugt et al., 2009), therefore, the correlation between condensation of energy levels and the cavity effect need to be carefully articulated. This particular finding supports our CEES observation that band structures of tubulin and microtubule are the same. The tubulins couple inside microtubule synchronously alleviating the noise or excess energy injected into it and at the same time, coupling induced energy level condensation defines the property of a single tubulin as the property of entire microtubule (except the relaxation time). Raman spectrum underpins the molecular origin of synchrony in tubulin and microtubule. Particular vibrational peaks of tubulin (Audenaert et al., 1889) survive in microtubule (Fig. 2b and c) along with the new atomic vibrations characteristic of a microtubule. The additional vibrational modes of microtubule are related to the elastic string properties. The elastic atomic residues located in the tubulins and distributed all over the microtubule oscillate in harmony to transport and drain out excess energy; this contributes to identical energy transmissions in the CEES spectrum.

In the 2D surface Raman profile of a single microtubule (Fig. 2d), particular vibrational frequency is assigned a particular color, thus, localizations of two similar colored dots, red and green exhibit the convergence of vibrational energy into two particular regions. Thus, the distribution of red and green dots along the microtubule length confirms that the vibrations surviving for pico-seconds and nanoseconds are homogeneously distributed all over the length of the microtubule, tubulins are coherently vibrating. Finally, in Fig. 2(e) a

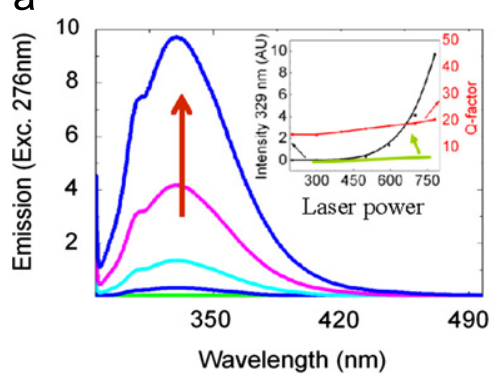

b

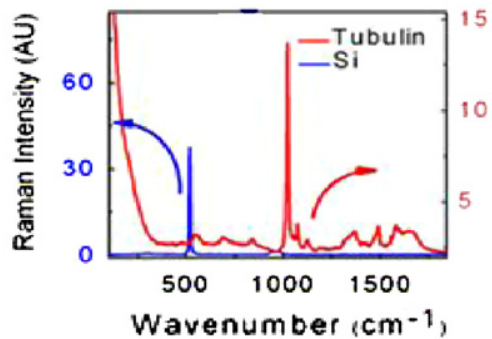

C

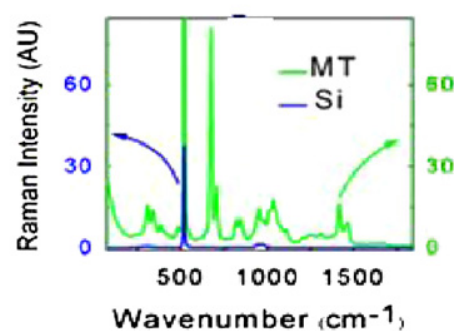

d

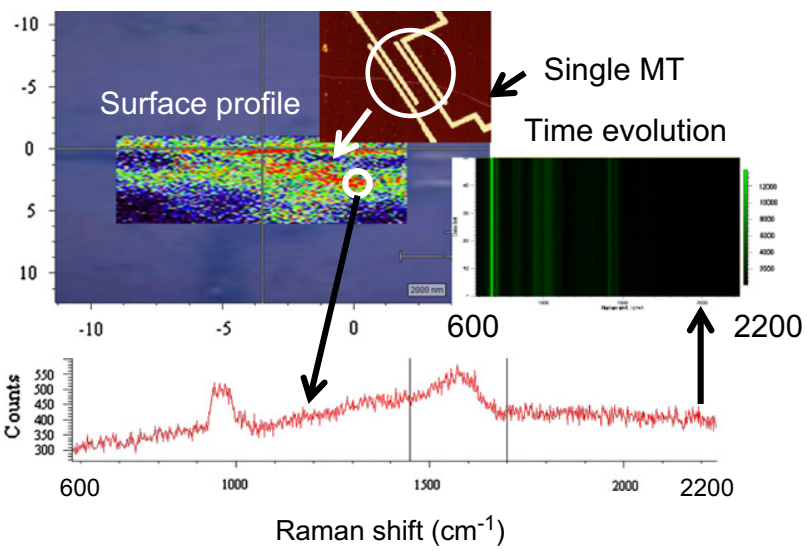

e

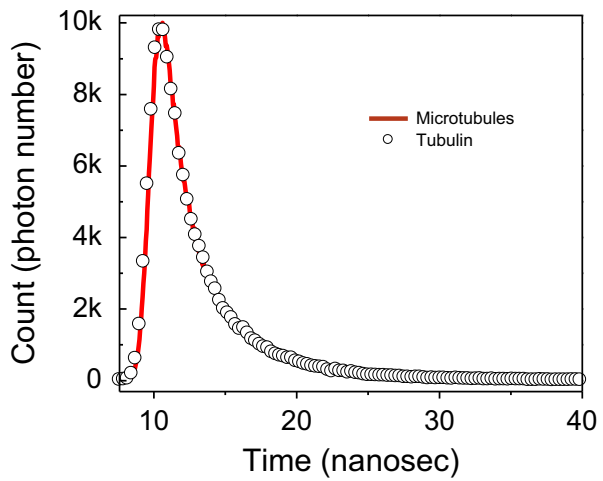

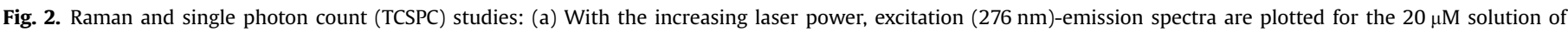

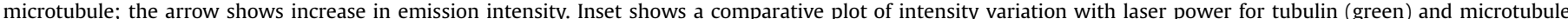

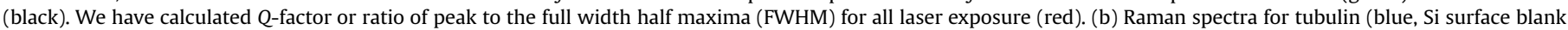

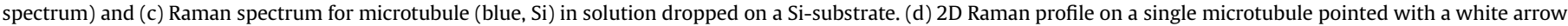

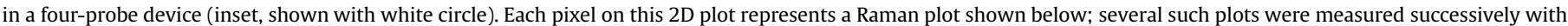

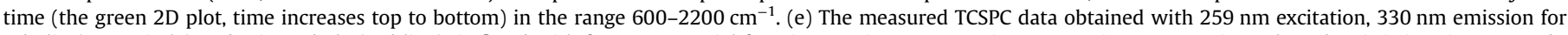

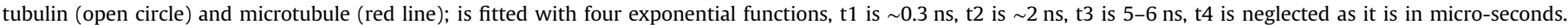
(For interpretation of the references to color in this figure legend, the reader is referred to the web version of this article.) 
a

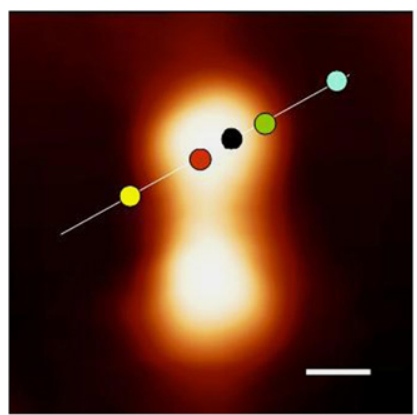

C

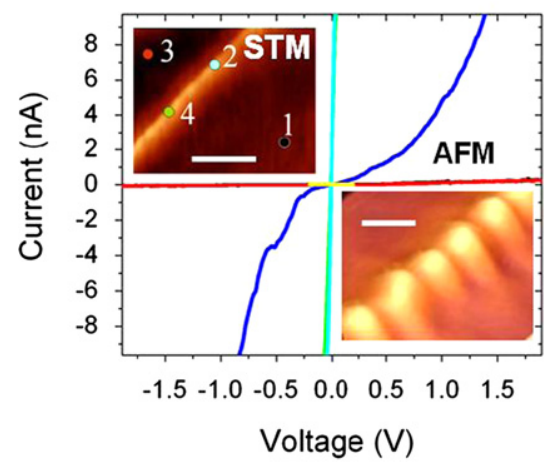

e

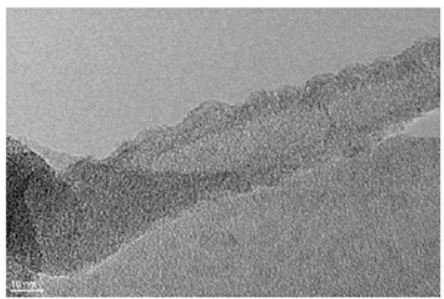

b

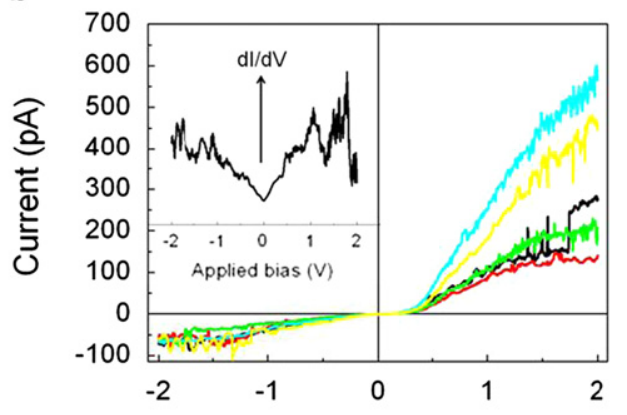

d

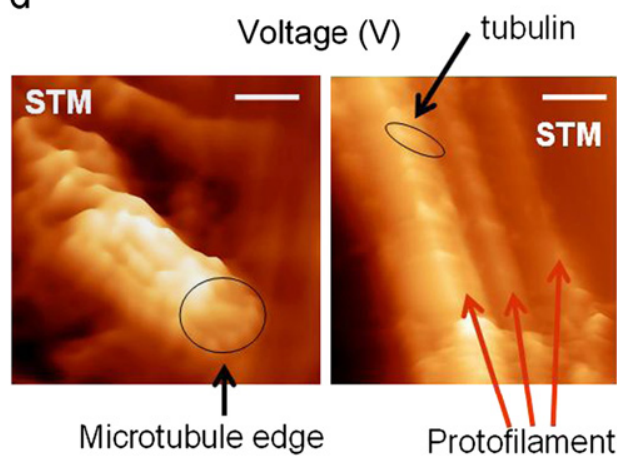

$f$

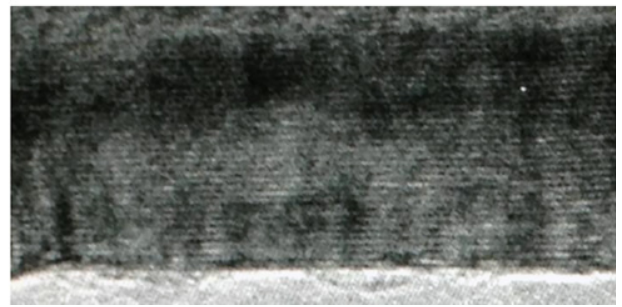

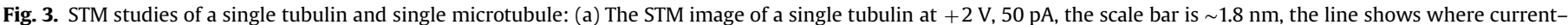

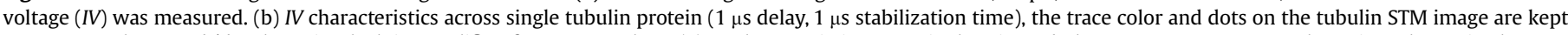

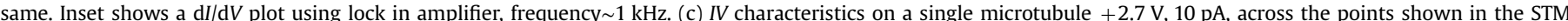

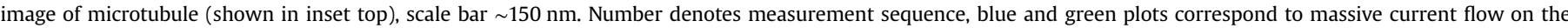

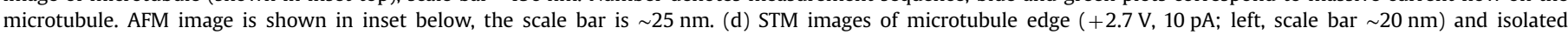

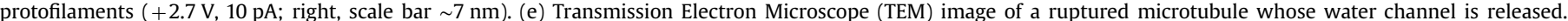

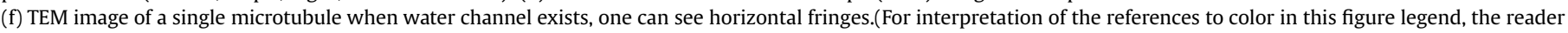
is referred to the web version of this article.)

time correlated single photon counts (TCSPC) were plotted for microtubule and tubulin protein to find that the relaxation times in the nano- and pico- second domains are also identical. During optical measurements in solution microtubule length was approximately $1-2 \mu \mathrm{m}$, since $1 \mu \mathrm{m}$ long microtubule has 1625 tubulin dimers, hence experimental evidences confirm that even after assembling into microtubule the energy levels and the relaxation behavior of tubulin protein do not change.

\subsection{Tunneling image and atomic resolution study of single tubulin and single microtubule with and without water}

We begin the electronic study with tubulin protein imaging. Only HOPG isolates single proteins during adsorption. Sometimes, two single protein dimers are paired; by rotating the STM tip-scan angle (Fig. 3a) tubulins were separated. To measure the electronic property, tubulin molecules were immobilized on the HOPG substrate at $77 \mathrm{~K}$. As standard protocol, the current voltage characteristic (IV) was first measured on the HOPG substrate, then on the single tubulin molecule and finally the STM tip was moved back to the substrate and measured $I V$ again. The cyclic process was repeated to confirm reproducibility. Fig. 3(a,b) shows how $I V$ is measured along the length of a single tubulin dimer to find that the central region of the dimer suppresses the conductivity significantly. This is an interesting observation, while studying single molecules it is always observed (Bandyopadhyay et al., 2006, 2010; Bandyopadhyay and Wakayama, 2007) that the tunneling conductivity between the substrate and the STM tip is less than that measured across the molecule. However, this is just opposite for the tubulin protein. Since, to the best of our knowledge this is the first report of electronic property for a single protein unit, therefore, it is not possible to confirm at this point, whether this is a general feature of the proteins or it is typical for the tubulin protein. However, in spite of insulation by $4 \mathrm{~nm}$ tubulin the remarkable electronics of $25 \mathrm{~mm}$ wide microtubule (Pizzi et al., 2011; Priel et al., 2006) is a crucial transformation since one should not expect any tunneling at all.

The IV of single tubulin molecule suggests that it is insulating, but current increases linearly with the increasing bias. Since 
linearity suppresses the normal exponential behavior, it suggests filtering of the current by the single protein molecule. For the ac measurement, the STM feedback loop is switched off (Oulton et al., 2008), using external circuit single tubulin ac conductivity is measured, which is much lower than the dc conductivity. The differential $\mathrm{d} I / \mathrm{d} V$ output shows multiple non-linear current behaviors in the inset of Fig. 3b, reflecting its capacitive storage properties. By varying the tip bias during STM scanning it is seen that the contrast changes homogeneously over $\alpha$ and $\beta$ tubulins, therefore, the charge that constitutes tubulin potential is always homogeneously delocalized all over the protein structure.

To get microtubule we reconstitute Porcene brain neuron extracted tubulins as described in Section 3. An extensive AFM and STM measurements are carried out on the $\mathrm{SiO}_{2}$ and HOPG surfaces respectively. Single tubulin protein is an insulator but single microtubule is 1000 times more conducting as shown in Fig. 3c. To understand the reason, the UHV condition is sustained at $77 \mathrm{~K}$ as water molecules are released from the microtubule core-a typical end part of a water-extracted microtubule is shown in Fig. 3d. Waterextracted microtubule behaves like an insulator as shown in Fig. 3d (right), therefore, the interstitial water channel inside microtubule is solely responsible for the 1000 times more conductivity than tubulin. The large tunneling current across $25 \mathrm{~nm}$ wide microtubule (insulator) is not via tubulin-water-tubulin route, the water core should act as a current source by storing charges. Dried microtubule does not show energy levels identical to tubulin protein, therefore, the water channel holds the proteins in a mechanism that does not allow splitting of energy levels of tubulins.

Microtubule solution is dropped on the $\mathrm{SiO}_{2}$ substrate and four electrodes are grown on top of it as shown in Fig. 4a so that the longitudinal conductivity and other electronic measurements are carried out (Makarovski et al., 2007; Samitsu et al., 2005; Walton et al., 2007; Zheng et al., 2004). During four probe measurements since current is sent from outer two electrodes and voltage drop is measured across the two central electrodes, the $300 \mathrm{M} \Omega$ contact resistance is nullified, and the measured resistance drops below $1 \mathrm{M} \Omega$ (Park, 2011). To understand the localized density of states on the microtubule, it is imaged at $77 \mathrm{~K}$ at different tip biases to find that electron density (Bandyopadhyay and Acharya, 2008; Smith et al., 1990) is homogeneously distributed over the entire protofilament and any induced potential fluctuation is delocalized all over the length (Fig. 3d (right)). The delocalization feature is responsible for four-probe unique conductivity, and automated noise management; again, delocalization disappears if water channel is removed. Then, at a higher bias $>2 \mathrm{~V}$, proto-filaments disintegrate in one scan. In the atomic force microscope (AFM) measurement, protofilaments do not break apart. In the AFM images, only helical tubulin rings are visible, while STM images show only longitudinal protofilaments, when water is inside, otherwise, it is a disintegrated mass of proteins. This suggests that the water channel (Fig. $3 \mathrm{c}$ inset) controls microtubule's internal conductivity and force modulation. In TEM, both helical ring and longitudinal fringes due to the water channel are visible, if water channel is released the fringes disappear and rings split (Fig. 3e, f).

\subsection{Four-probe electrode based electronic measurement of single microtubule device}

The challenges associated with the microtubule's IV measurement are discussed in the supporting online materials (Minoura and Muto, 2006). Here we compare two cases (i) the device is earthed and (ii) under floating condition, which estimates the amount of charge storage at the junction. Microtubule exhibits a perfectly square hysteresis behavior (Fig. 4a,b) (Damjanovic, 2006), it means the dipole moments of tubulin proteins rotate synchronously by $\pm 23^{\circ}$, which plays a vital role in switching the conductivity or memory states. The hysteresis area increases with the maximum applied bias during an $I V$ measurement, however, the flat region does not disappear, and the flatness suggests an alleviation of noise. The flatness originates during normalization, the raw data suggest a small-angle slope in $I V$. Fig. 4c shows that the square-nature survives even under extreme noise. However, when a current source is used to measure $I V$, the device does not show the square $I V$ feature; beyond a certain input current, the

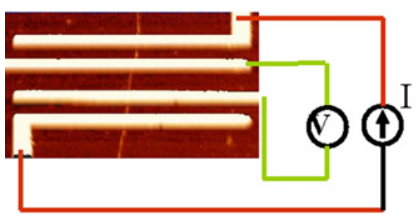

b

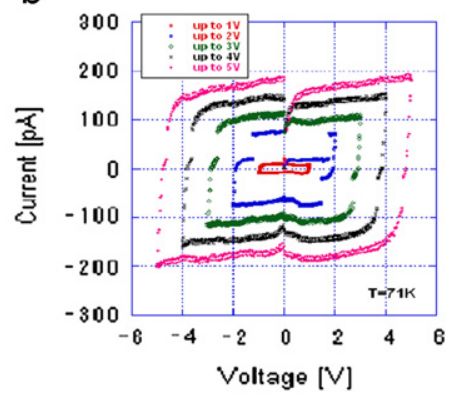

C

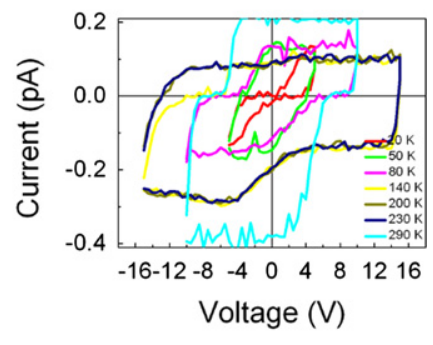

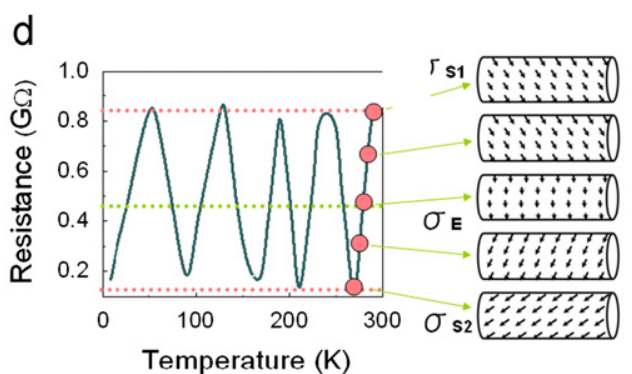

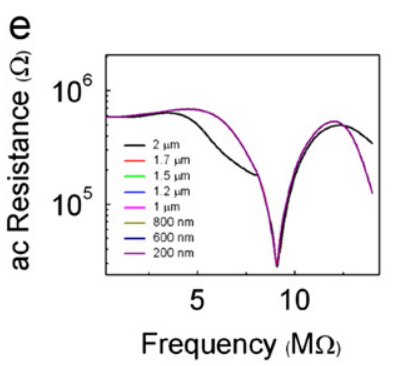

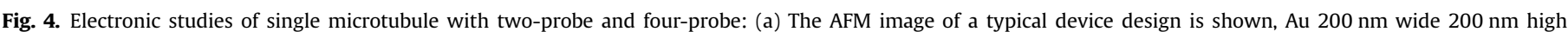

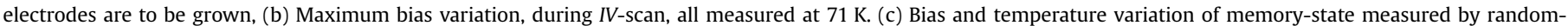

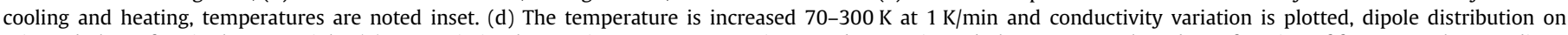

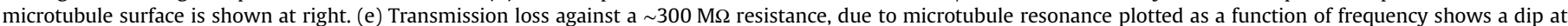
$9.8 \mathrm{M} \Omega$. 
voltage output varied linearly, thus, the microtubule develops a unique polarization beyond a threshold charge injection. The dielectric studies on single microtubule device suggest that microtubule has extremely large charge density $4.3 \mathrm{e} / \AA$ (Sanab ria, 2005). This value is $10^{6}$ times more than the conventional inorganic semiconductors and $10^{2}$ times lower than any metal, therefore, classifying microtubule as metal, insulator or semiconductor is incorrect (for the details of single microtubule conductivity measurement, see supporting information online). Thermal noise flips the encoded conductivity of a single microtubule rand omly, however, above $0.1 \mathrm{~K} / \mathrm{min}$ heat injection, the conductivity oscillates as shown in Fig. 4d. Thus, microtubule's automated noise alleviation has a limit, under an extreme noise it is programmed to oscillate around the encoded conductivity.

\subsection{Automated noise alleviation and ac response of single microtubule device}

The most critical challenge to study ac response (Egard et al., 2010; He et al., 2008) of a nanotube is the normalization (see Fig. S2(a-e) online, detailed ac characterization is discussed online). Fig. 4e shows that even ac power transmission is independent of the length of the microtubule. Since proteins have incredibly large number of transmission-channels and only a few respond under typical measurements. Accurate identification required measuring the same resonance peaks via different experimental setups, thus, statistically dominant peaks were isolated as the most probable resonance peaks by checking thousands of noise and actual peaks one by one. The measurement of microtubule ac resonance requires a shielded environment as shown in Fig. 5a. Three distinct circuits were used as shown in Fig. 5b-e. Fig. 5b shows a circuit that sends ac signal to the microtubule and then measures the dc resistance loss, the frequency causing sharp increase in conductivity is the resonance peak. Using Fig. 5c circuit, the transmittance and reflectance of microtubule are plotted between $1 \mathrm{KHz}$ and $20 \mathrm{GHz}$, therein at particular frequencies, the transmittance is large. At these particular frequencies, the microtubule sends ac signal with almost no resistance (much less than $0.04 \Omega$ ). In Fig. 5d, e the transmittance measurement is repeated, however, the ac input signal is bifurcated using paired electrodes to induce an additional capacitive effect, so that the contact effects of pseudo capacitive and inductive elements are nullified. Three circuit outputs are shown in Fig. 5f, the common peaks are taken into account as absolute resonance peak. Note that there are several harmonics for each peak and if water channel is released, no resonance peak is observed for the single microtubule.

\subsection{Multiple comparative measurements of resonance band of single microtubule}

With the eight primary resonance peaks, the microtubule is truly a vibrating resonant string (Jelínek et al., 1999; Jelínek and Pokorný, 2001) and this vibration survived when we dipped microtubules in serum, hence living cells will exhibit these features. Microtubule develops a positive and a negative polarity, and automatically generates a potential gradient across its length, as a result, its resistance differs if we use positive or negative voltage to measure conductivity (Tran et al., 1997). In trillions of cells inside our body, this particular electric field gradient along with autto-watt power ( $1 \mathrm{fA}, 90 \mathrm{mV}$ ) drive microtubules in a complex pattern to execute several tasks, an additional $\mathrm{MHz}$ source could externally tune the dynamic instability, which is the signature of several diseases. From the comparative study of tubulin protein and single microtubule, it is evident that if the nature changes only one property of a single tubulin, the property of entire microtubule would change. It enables nature to add a particular single molecule to tubulin to create cells of a species that survive $-30{ }^{\circ} \mathrm{C}$ or change the doped molecule to enable it surviving at $60{ }^{\circ} \mathrm{C}$ for other species, and this practice is visible in the plant, animal, fungi and Protista kingdom, but remained unnoticed. Microtubule is a generic platform in which nature has synthesized peculiarities of robust species living around us.

\section{Experimental section (details in the supporting online material)}

Microtubules are extracted from Porcine's brain by Cytoskeleton (Denver, CO), we purchased tubulin protein including all associated tubulin-to-microtubule conversion kits, and reconstituted microtubule in our laboratory. Purified microtubule subunits (tubulins) were preserved at $-80^{\circ} \mathrm{C}$. To polymerize tubulin (Borisy et al., 1975; Fygenson et al., 1994), into $6.5 \mu \mathrm{m}$ long microtubules, $160 \mu \mathrm{l}$ of Microtubule cushion buffer $(60 \% \mathrm{v} / \mathrm{v}$ glycerol, $80 \mathrm{mM}$
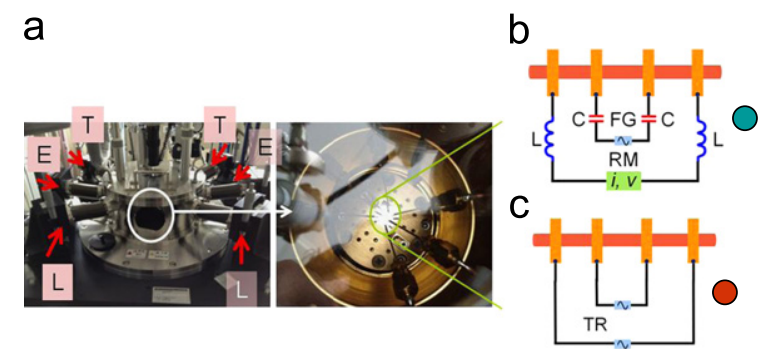

d

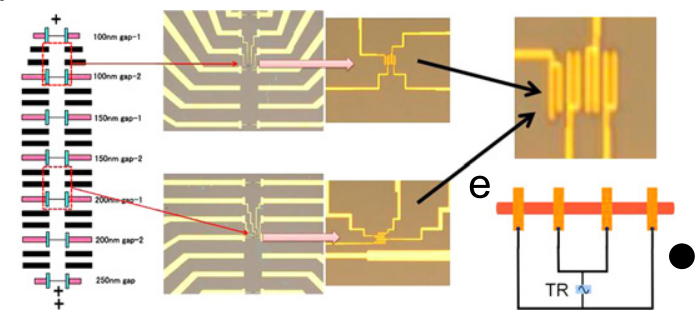

f

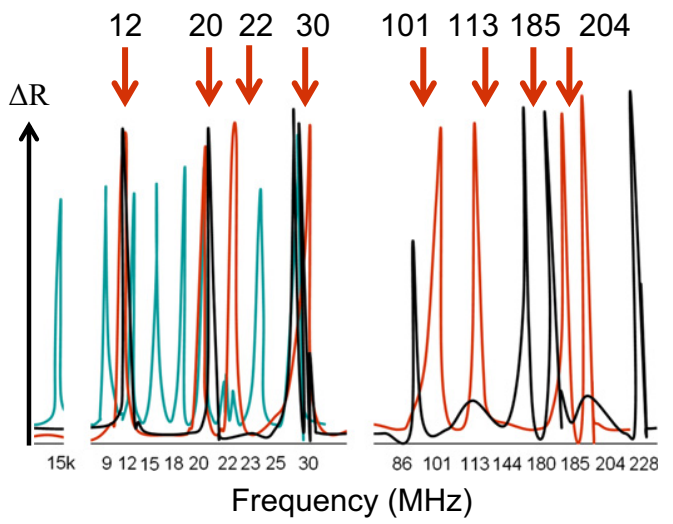

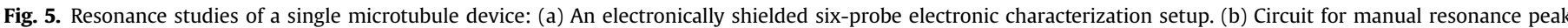

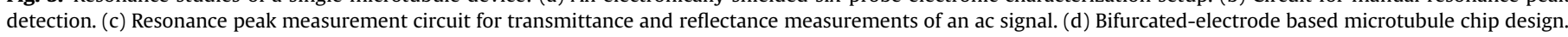

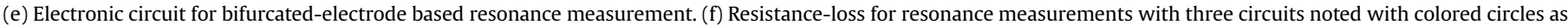
a function of frequency measured from $1 \mathrm{kHz}$ to $1.3 \mathrm{GHz}$. Statistically most-occurred peaks are noted above. 
PIPES pH 6.8, $1 \mathrm{mM}$ EGTA, $1 \mathrm{mM} \mathrm{MgCl}_{2}$ ) was added to $830 \mu \mathrm{l}$ of general tubulin buffer ( $80 \mathrm{mM}$ PIPES pH 7, $1 \mathrm{mM}$ EGTA, $2 \mathrm{mM}$ $\mathrm{MgCl}_{2}$ ) and $10 \mu \mathrm{l}$ of $100 \mathrm{mM}$ GTP solution. This mixture is kept in an ice bath for $10 \mathrm{~min}$. From this mixture, $200 \mu \mathrm{l}$ solution is added to $1 \mathrm{mg}$ of tubulin and again it is incubated in an ice bath for $10 \mathrm{~min}$. Afterwards this stock is placed in an incubator at $35-37{ }^{\circ} \mathrm{C}$ for $40 \mathrm{~min}$. Now, to stabilize microtubules $20 \mu \mathrm{l}$ of Paclitaxal dissolved in anhydrous DMSO is added to the solution and it is incubated for a further $10 \mathrm{~min}$ at $37^{\circ} \mathrm{C}$. The microtubule length is tuned $\sim 4-20 \mu \mathrm{m}$.

To prepare film, the solution is diluted 8 times using microtubules cushion buffer and paclitaxol DMSO solution and dropped on a $45^{\circ}$ tilted $\mathrm{Si}(100)$ substrate and interdigited electrodes were kept on a $-20^{\circ} \mathrm{C}$ bath overnight. An electric field is applied across the substrate for parallel alignment of the microtubules. The excess microtubule solution is removed from the substrate using a filter paper (Whatman), this is the best route to carry out STM and AFM studies. Now the substrate is dipped into General Tubulin Buffer and once again dried blowing $\mathrm{N}_{2}$ in the similar fashion. The process is repeated twice. The substrate is placed in a refrigerator for $3 \mathrm{~h}$ to dry out the surface partially. Reconstitution of $\alpha$ and $\beta$ tubulin into $\alpha \beta$ heterodimer of dimensions $46 \times 80 \times 65 \AA^{3}$ is confirmed via UHV-AFM and UHV-STM with an atomic sharp tip that has $0.01 \AA$ tip, we also confirmed the 13 proto-filaments in the microtubules produced by Raman and STM/AFM imaging.

\section{Conclusion}

We have studied Combined Excitation Emission Spectroscopy (CEES) and Raman for single tubulin protein, microtubule nanowire with and without water to find that the emission peaks in the CEES plot as well as nano-seconds decay profile of fluorescence are identical for isolated tubulin protein and the microtubule nanowire. Using AFM attached tip-enhanced Raman spectroscopy we have determined that only a particular vibrational mode of the microtubule is populated. These three results suggest that the microtubule's optical and thermal vibrations are programmed inside a single tubulin dimer, and that is eventually reflected when we measure the single microtubule of any particular length. To advance our conclusion further, we studied single protein molecule and microtubule using STM and found that $25 \mathrm{~nm}$ wide microtubule is more conducting than the $4 \mathrm{~nm}$ wide single tubulin, which is significant. If the water channel is released, the microtubule becomes an insulator once again, thus, the water channel controls the conductivity of the microtubule. If we combine two conclusions noted above, water channel and protein molecule together control the emergent properties of the material, the only possible relation that could couple water molecule and protein is an electromagnetic resonant oscillation, so we measured ac resonance properties to find a large number of resonance peaks for the microtubule. In the future, we study these resonance peaks for wireless communication, coherence and synchrony, to understand the information processing in the brain and in the living cell, to unravel a world beyond chemicalonly-biology.

\section{Author Contributions}

A.B designed research; S.S designed and built the microtubule device; S.S, A.B, K.H and S.G performed the experiments; A.B and S.S analyzed the data; A.B wrote the paper and D.F reviewed the work.

\section{Acknowledgment}

The authors acknowledge Eiichiro Watanabe and Daiju Tsuya of Nanotechnology Innovation Station, NIMS Sengen-site Nano-foundry sponsored by Ministry of Science, Education, Culture and Sports (MEXT), Govt. of Japan. The current research work is funded by the Asian office of Aerospace R\&D, Govt. of USA FA2386-11-10001AOARD104173 and FA2386 -10-1-4059 AOARD-10-4059.

\section{Appendix A. Supporting information}

Supplementary data associated with this article can be found in the online version at http://dx.doi.org/10.1016/j.bios.2013.02.050.

\section{References}

Ahmad, F.J., Joshi, H.C., Centonze, V.E., Baas, P.W., 1994. Neuron 12, 271-280. Agarwal, R., Barrelet, C.J., Lieber, C.M., 2005. Nano Letters 5, 917-920.

Audenaert, R., Heremans, L., Heremans, K., Engelborghs, Y., 1889. Biochimica et Biophysica Acta-Protein Structure and Molecular Enzymology 996, 110-115. Barabási, A.L., Albert, R., 1999. Science 286, 509-512.

Butts, C.T., 2009. Science 325, 414-416.

Braun, H.A., Voigt, K., Huber, M.T., 2003. Biosystems 71, 39-50.

Bandyopadhyay, A., Miki, K., Wakayama, Y., 2006. Applied Physics Letters 89, 243503-243506

Bandyopadhyay, A., Sahu, S., Fujita, D., Wakayama, Y., 2010. Physical Chemistry Chemical Physics 12, 2198-2208.

Bandyopadhyay, A., Wakayama, Y., 2007. Applied Physics Letters 90, 023512-023514.

Borisy, G., Marcum, J., Olmsted, J., Murphy, D., Johnson, K., 1975. Annals of the New York Academy of Sciences 253, 107-132.

Bandyopadhyay, A., Acharya, S., 2008. Proceedings of the National Academy of Sciences of the United States of America 105 (2008), 3668-3672.

Carlier, M., Melki, R., Pantaloni, D., Hill, T., Chen, Y., 1987. Proceedings of the National Academy of Sciences of the United States of America 84, 5257-5261.

Damjanovic, D., 2006. The Science of Hysteresis, vol. 3, pp. 337-465.

Dekker, C., Ratner, M.A., 2001. Physics World 14, 29-33.

Dierolf, V., O'Donnell, K., 2010. Rare earth doped III-nitrides for optoelectronic and spintronic applicationsTopics in Applied Physics, vol. 124. Springer, The Netherlands, pp. 221-268.

Dierolf, V., Koerdt, M., 2000. Physical Review B 61, 8043-8052.

Egard, M., Johansson, S., Johansson, A.C., Persson, K.M., Dey, A., Borg, B., Thelander, C., Wernersson, L.E., Lind, E., 2010. Nano Letters 10, 809-812.

Fygenson, D.K., Braun, E., Libchaber, A., 1994. Physical Review E 50, 1579-1588.

Fink, H.W., Schönenberger, C., 1999. Nature 398, 407-410.

Grigor'kin, A., Dunaevskiı̆, S., 2007. Physics of the Solid State 49, 585-590.

Gerhart, J., Kirschner, M., Moderbacher, E.S., 1997. Cells, Embryos, and Evolution. Blackwell Science, Malden, MA.

He, R., Feng, X., Roukes, M., Yang, P., 2008. Nano Letters 8, 1756-1761.

Jelínek, F., Pokorný, J., 2001. Electromagnetic Biology and Medicine 20, 75-80.

Jelínek, F., Pokorný, J., Šaroch, J., Trkal, V., Hašek, J., Palán, B., 1999. Bioelectrochemistry and Bioenergetics 48, 261-266.

Moriya, S., Tanaka, K., Ohkuma, M., Sugano, S., Kudo, T., 2001. Journal of Molecular Evolution 52, 6-16.

Mange, D., Tomassini, M., 1998. Bio-inspired Computing Machines: Towards Novel Computational Architectures. Presses polytechniques et universitaires romandes, Lausanne.

Mesquita, M.V., Vasconcellos, A.R., Luzzi, R., 1993. Physical Review E 48, 4049-4059.

Moskalenko, S., Miglei, M., Khadshi, P., Pokatilov, E., Kiselyova, E., 1980. Physics Letters A 76, 197-200.

Michalski, P., Mele, E.J., 2008. Physical Review B 77, 085429.

Makarovski, A., Zhukov, A., Liu, J., Finkelstein, G., 2007. Physical Review B 76, R161405.

Minoura, I., Muto, E., 2006. Biophysics Journal 90, 3739-3748.

Nielsen, M., Gadagkar, S., Gutzwiller, L., 2010. BMC Evolutionary Biology 10, 113.

Nielsen, M.G., Caserta, J.M., Kidd, S.J., Phillips, C.M., 2006. Evolution and Development 8, 23-29.

Oulton, R.F., Sorger, V.J., Genov, D., Pile, D., Zhang, X., 2008. Nature Photonics 2, 496-500.

Prodan, E., Prodan, C., 2009. Physical Review Letters 103, 248101.

Pizzi, R., Strini, G., Fiorentini, S., Pappalardo, V., Pregnolato M., 2011. Focus on Artificial Neural Networks cap. In: Flores John, A. (Ed.), ISBN: 978-1-61324-2858. Nova Science Publisher Inc., vol. 9, pp. 191-207.

Priel, A., Ramos, A.J., Tuszynski, J.A., Cantiello, H.F., 2006. Biophysics Journal 90, 4639-4643.

Park, H., 2011. Contact Limiting Effects of Nanowire Devices, Ph.D. Thesis, Rhode Island, Brown University.

Roberts, E., Magis, A., Ortiz, J.O., Baumeister, W., Luthey-Schulten, Z., 2011. PLOS Computational Biology 7, e1002010. 
Redeker, V., Frankfurter, A., Parker, S.K., Rossier, J., DetrichIII, 2004. Biochemistry 43, 12265-12274.

Rakitin, A., Aich, P., Papadopoulos, C., Kobzar, Y., Vedeneev, A., Lee, J., Xu, J., 2001. Physical Review Letters 86, 3670-3673.

Rotaru, V., Lajoie-Mazenc, I., Tollon, Y., Raynaud-Messina, B., Jean, C., Détraves, C., Julian, M., Moisand, A., Wright, M., 1999. Biology of the Cell 91, 393-406.

Storm, A., Van Noort, J., De Vries, S., Dekker, C., 2001. Applied Physics Letters 79, 3881-3883.

Shibata, T., Ueda, M., 2008. Biosystems 93, 126-132.

Szendro, P., Vincze, G., Szasz, A., 2001a. European Biophysics Journal 30, 227-231.

Szendro, P., Vincze, G., Szasz, A., 2001b. Electromagnetic Biology and Medicine 20, 215-229.

Song, C., Havlin, S., Makse, H.A., 2005. Nature 433, 392-395.

Strogatz, S.H., 2001. Nature 410, 268-276.

Sipper, M., 2002. Machine Nature: The Coming Age of Bio-inspired Computing. McGraw-Hill, New York.

Sahu, S., Ghosh, S., Fujita, D., Bandyopadhyay, A., 2011. Journal of Computational and Theoretical Nanoscience 8, 509-515.
Sanabria, H., 2005. Impedance Spectroscopy of Polyelectrolytes: Case Study of Alpha-Beta Tubulin Suspensions, Ph.D. Thesis, University of Houston.

Smith, D., Hörber, J., Binnig, G., Nejoh, H., 1990. Nature 344, 641-644.

Samitsu, S., Shimomura, T., Ito, K., Fujimori, M., Heike, S., Hashizume, T., 2005. Applied Physics Letters 86, 233103.

Tran, P., Walker, R., Salmon, E., 1997. Journal of Cell Biology 138, 105-117.

Teuscher, C., Mange, D., Stauffer, A., Tempesti, G., 2003. Biosystems 68, 235-244. van Vugt, L.K., Zhang, B., Piccione, B., Spector, A.A., Agarwal, R., 2009. Nano Letters 9, 1684-1688.

Walton, A., Allen, C., Critchley, K., Górzny, M.t., Brydson, R., Hickey, B., Evans, S., 2007. Nanotechnology 18, 065204.

Zhang, D., Gao, Z., 2012. Robotics and Computer-Integrated Manufacturing 28, 484-492.

Zhang, Y., Austin, R., Kraeft, J., Cox, E., Ong, N., 2002. Physical Review Letters 89, 198102.

Zheng, G., Lu, W., Jin, S., Lieber, C.M., 2004. Advanced Materials 16, 1890-1893. 